\title{
Erratum to: FUGE: A joint meta-heuristic approach to cloud job scheduling algorithm using fuzzy theory and a genetic method
}

\author{
Mohammad Shojafar • Saeed Javanmardi • \\ Saeid Abolfazli • Nicola Cordeschi
}

Published online: 19 March 2015

(C) Springer Science+Business Media New York 2015

\section{Erratum to: Cluster Comput \\ DOI 10.1007/s10586-014-0420-x}

The original version of this article unfortunately contained mistakes which are listed below. The authors apologise for the errors and the inconvenience caused to the readers.

On page 9, the first line of the paragraph above Eq. (3.1) should have read as "We formulate a linearly constraint programming model for" instead of "We formulate a linear programming model for".

On the same page, in the first line of the last paragraph, the word "concave" should have read as "convex". On page 10 , in the paragraph that starts as "According to" the words "(linear)" and "use maximize instead of minimization and" should not have appeared. The word "concave" should have read as "convex".

The online version of the original article can be found under doi:10.1007/s10586-014-0420-x.

M. Shojafar $(\varangle) \cdot$ N. Cordeschi

Department of Information Engineering Electronics and

Telecommunications (DIET), University Sapienza of Rome,

via Eudossiana 18, 00184 Rome, Italy

e-mail: m.shojafar@yahoo.com; shojafar@diet.uniroma1.it

URL: http://www.mshojafar.com

N. Cordeschi

e-mail: cordeschi@diet.uniroma1.it

S. Javanmardi

Research and Education center, Nikan network Company,

Shiraz, Fars, Iran

e-mail: info@nikannetwork.com; saeedjavanmardi@gmail.com

S. Abolfazli

Center for Mobile Cloud Computing, University of Malaya,

Kuala Lumpur, Malaysia

e-mail: Abolfazli@ieee.org
Therefore, the paragraph should have read as "According to Eq. (4), $f$ is order one for $x$ and $y$, so we can easily calculate Hessian matrix for evaluating convexity. Therefore, we have in following:

$$
\begin{aligned}
f(x, y) & =\frac{C}{x}+\frac{D}{y}, \\
f_{x}^{\prime} & =-C / x^{2} \leq 0, \quad f_{y}^{\prime}=-D / y^{2} \leq 0, \\
f_{x x}^{\prime \prime} & =+2 C / x^{3}, \quad f_{y y}^{\prime \prime}=+2 D / y^{3}, \\
f_{x y}^{\prime \prime} & =f_{y x}^{\prime \prime}=0, \\
\text { Det.: } & =f_{x x}^{\prime \prime} f_{y y}^{\prime \prime}-f_{x y}^{\prime \prime} f_{y x}^{\prime \prime}=\frac{4 C D}{x^{3} y^{3}} \geq 0
\end{aligned}
$$

where Eq. (5) is the Hessian Determinant for the objective function (4). Therefore, objective function is convex and for the practical implementation with the fuzzy method we chose to maximize the opposite of the $f$ instead of minimization by applying Karush-Kuhn-Tucker (KKT) [6]." 